\title{
Oxidative stress and nitric oxide signaling related biomarkers in patients with pulmonary hypertension: a case control study
}

Shuai Zhang ${ }^{1,2}$, Ting Yang ${ }^{1,2}$, Xiaomao Xu ${ }^{3,4}$, Meng Wang ${ }^{5}$, Linye Zhong ${ }^{1,2}$, Yuanhua Yang ${ }^{1,2}$, Zhenguo Zhai ${ }^{1,2}$, Fei Xiao ${ }^{4,6}$ and Chen Wang ${ }^{4,7,8^{*}}$

\begin{abstract}
Background: Oxidative stress (OS) and reduced nitric oxide (NO) bioavailability contribute to the pathogenesis of pulmonary hypertension (PH). Whether there are associations between OS and NO signaling biomarkers and whether these biomarkers are associated with the severity of $\mathrm{PH}$ remain unclear.

Methods: Blood samples were collected from 35 healthy controls and 35 patients with pulmonary arterial hypertension (PAH, $n=12$ ) or chronic thromboembolic pulmonary hypertension ( $C T E P H, n=23)$. The mean pulmonary artery pressure (mPAP) and pulmonary vascular resistance index (PVRI) were measured by right heart catheterization. We measured the derivative of reactive oxygen molecules (d-ROMs), biological antioxidant potential (BAP) and superoxide dismutase (SOD) by automatic biochemical analyzer, malondialdehyde (MDA) and asymmetric dimethylarginine (ADMA) by enzyme-linked immunosorbent assay. The relationship between oxidative-antioxidative biomarkers and ADMA, as well as their association with pulmonary hemodynamics, were analyzed.

Results: Compared with age- and gender-matched controls, there was no significant difference of $d$-ROMs in PAH and CTEPH patients; MDA was increased in CTEPH patients ( $P=0.034) ; B A P$ and SOD were decreased in PAH $(P=0.014$, $P<0.001)$ and $C T E P H$ patients $(P=0.015, P<0.001)$; ADMA level was significantly higher in $P A H(P=0.007)$ and $C T E P H$ patients $(P<0.001)$. No association between oxidative-antioxidative biomarkers and ADMA was found. Serum ADMA concentration was correlated with mPAP $(r=0.762, P=0.006)$ and PVRI $(r=0.603, P=0.038)$ in PAH patients.

Conclusions: The antioxidative potential and $\mathrm{NO}$ signaling are impaired in $\mathrm{PAH}$ and $\mathrm{CTEPH}$. Increased serum ADMA level is associated with unfavorable pulmonary hemodynamics in PAH patients. Thus, ADMA may be useful in the severity evaluation and risk stratification of PAH.
\end{abstract}

Keywords: Oxidative stress, Nitric oxide, Pulmonary hypertension, Biomarkers

\section{Background}

Pulmonary hypertension (PH) is a complex pulmonary vascular disease caused by different reasons, such as chronic hypoxia, left heart diseases, chronic respiratory diseases and thromboembolism [1]. In the classification of PH by World Health Organization (WHO), pulmonary arterial hypertension (PAH) and chronic thromboembolic pulmonary hypertension (CTEPH) share the pathological

\footnotetext{
* Correspondence: cyh-birm@263.net

${ }^{4}$ National Clinical Research Center of Respiratory Diseases, Beijing, People's Republic of China

7Department of Respiratory Medicine, Capital Medical University, Beijing, People's Republic of China

Full list of author information is available at the end of the article
}

characteristic of vascular remodeling. Pulmonary vascular remodeling is characterized by medial hypertrophy, intimal proliferative and fibrotic changes, adventitial thickening, and thrombotic lesions [1]. Although the mechanisms involved are incompletely understood, endothelial dysfunction with proliferation of endothelial cells and smooth muscle cells plays an important role in vascular remodeling [2]. One character of endothelial dysfunction is oxidative stress (OS) [3,4], which can increase the production of reactive oxygen species (ROS) and reactive nitrogen species (RNS) and decrease the bioavailable nitric oxide (NO).

\section{() Biomed Central}


All vascular cells, including endothelial cells, smooth muscle cells, and adventitial cells, can produce ROS. In patients with vascular diseases, the oxidative-antioxidative balance in the vessel wall is compromised due to the increase of ROS production by these cells, sometimes coupled with decreased antioxidant defense. ROS may trigger signals that further exacerbate smooth muscle hypercontractility, endothelial barrier dysfunction, and vascular remodeling [5], Increasing evidence demonstrates that OS plays a contributory role in the pathogenesis of PAH [6-9]. Recently, increased markers of OS have been found in various animal models, such as C57/BL6 mice exposed to hypobaric hypoxic conditions and Sprague-Dawley rats with monocrotaline-induced $\mathrm{PAH}$ $[10,11]$. However, few studies have explored the roles of oxidative-antioxidative biomarkers in $\mathrm{PH}$ patients.

$\mathrm{NO}$ is synthesized in the endothelium from L-arginine by NO synthase. Asymmetric dimethylarginine (ADMA) is an endogenous NO synthase inhibitor, which has been implicated in the pathogenesis of various cardiovascular diseases [12-16]. ADMA plasma levels are significantly elevated in idiopathic pulmonary arterial hypertension (IPAH), and correlated significantly with mixed-venous oxygen saturation, right atrial pressure, cardiac index, as well as survival $[17,18]$.

Whether oxidative-antioxidative biomarkers and ADMA are associated with mean pulmonary artery pressure (mPAP) and pulmonary vascular resistance index (PVRI) remains unclear. Both oxidative-antioxidative imbalance and impaired NO signaling are involved in endothelial dysfunction and vascular remodeling. NO relaxes smooth muscle and decreases its metabolism, which will reduce the production of ROS. Thus, there may be some association between ADMA and oxidative-antioxidative biomarkers, which need to be investigated to further understand the pathogenesis and pathophysiologic processes of $\mathrm{PH}$.

Therefore, we designed this case-control study to measure the oxidative-antioxidative biomarkers and ADMA level in the healthy controls, PAH patients, and CTEPH patients, with an attempt to identify the association between oxidative-antioxidative biomarkers and ADMA, simultaneously evaluate the association between these biomarkers and $\mathrm{PH}$ severity assessed by pulmonary hemodynamics. The level of these biomarkers was compared between PAH patients and their age- and gender-matched controls, between CTEPH patients and their age- and gender-matched controls respectively. The oxidative biomarkers we assessed included derivative of reactive oxygen molecules (d-ROMs) and malondialdehyde (MDA). Biological antioxidant potential (BAP) and superoxide dismutase (SOD) were assessed as the antioxidative biomarkers. d-ROMs and BAP, which are general evaluation of oxidative and antioxidative status respectively, have been used in the evaluation of disease severity and therapeutic effect in chronic obstructive pulmonary disease [19], idiopathic pulmonary fibrosis [20] and other diseases. ROS degrade polyunsaturated lipids, forming MDA, which is used as a biomarker to measure the level of $\mathrm{OS}$ in an organism [21,22]. SOD out-competes damaging reactions of superoxide, protects the cell from superoxide toxicity, and is widely used in scientific research and clinical practice $[23,24]$.

\section{Methods}

\section{Study population}

Consecutive adult patients newly diagnosed with PAH or CTEPH by pulmonary angiography and right heart catheterization were enrolled. $\mathrm{PH}$ has been defined as an increase in $\mathrm{mPAP} \geq 25 \mathrm{mmHg}$ [25]. PAH is characterized by the presence of pre-capillary PH, i.e., $\mathrm{mPAP} \geq 25 \mathrm{mmHg}$, pulmonary artery wedge pressure $\leq 15 \mathrm{~mm} \mathrm{Hg}$, and elevated pulmonary vascular resistance $>3$ Wood units, in the absence of other causes of pre-capillary $\mathrm{PH}$. The final diagnosis of CTEPH was based on the presence of precapillary $\mathrm{PH}$ in patients with multiple chronic/organized occlusive thrombi/emboli in the elastic pulmonary arteries. Patients were excluded if they had unstable atherosclerotic vascular disease, renal dysfunction, or untreated hyperlipidemia or if they were under treatment with nitrates, NO donors, prostaglandins, endothelin receptor antagonists, sildenafil, or antioxidant therapy. The demographic and clinical information, six minute walk distance (6MWD) and WHO functional class of all the patients was recorded. mPAP and PVRI were measured by right heart catheterization.

Age- and gender-matched healthy candidates were included as the control group for PAH group and CTEPH group, respectively. Healthy controls were selected from volunteers without any abnormality in the physical examination and laboratory tests. The exclusion criteria included: 1) with a history of respiratory disease, cardiac disease, cardiovascular and cerebrovascular disease, chronic liver and kidney failure, malignancy, diabetes mellitus, and/or any additional medical disorders; 2) smoking; and 3) using antioxidant drugs.

The study protocol was approved by the Ethics Committee of Beijing Hospital (2014BJYYEC-051-01). Informed consent was obtained from each subject.

\section{Blood samples}

Once the diagnosis was established, fresh blood samples were collected before the treatment except the basic treatment began. The basic treatment included diuretics, oxygen, cardiotonics and other supportive treatment. We used the separation gel coagulation promoting vacuum tubes. Within 1 hour after sample collection, the blood sample was centrifuged by $2280 \mathrm{~g}$ for 5 minutes. Then the serum was stored at $-80^{\circ} \mathrm{C}$. All the serum samples were 
measured simultaneously. Before the measurement of the biomarkers, serum samples were balanced to room temperature. Thawing and refreezing were avoided.

\section{Measurement of d-ROMs}

We measured d-ROMs level using the automatic biochemical analyzer (HITACHI 7600 Series, Japan). The method is based on the principle that in Tris- $\mathrm{HCl}$ buffer $(\mathrm{pH}=5)$, iron ions previously bonded to serum proteins can release and catalyze the conversion of serum hydroperoxides to alkoxyl and peroxyl radicals, which further react with chromogen, dimethyl paraphenylene diamine hydrochloride. Upon oxidation, its color becomes lighter, which is measured at $505 \mathrm{~nm}$. Tert butyl hydroperoxide (TBHP) was used as standard substance. The results were expressed as an equivalent of mmol TBHP Equiv/L.

\section{Measurement of BAP}

BAP level was measured using the automatic biochemical analyzer (AU 5400, Olympus, Japan). The assay is based on the ability of a colored ferric thiocyanate to decrease in absorption when $\mathrm{Fe}^{3+}$ ions are reduced to $\mathrm{Fe}^{2+}$. The absorbance is measured photometrically at $520 \mathrm{~nm}$ and calculating the amount of reduced ferric ions. Vitamin C (VitC) was used as standard substance. The results were expressed as an equivalent of mmol VitC Equiv/L.

\section{Measurement of SOD, MDA and ADMA}

SOD level was measured using the automatic biochemical analyzer (AU 5400, Olympus, Japan) and a commercial kit (Fujian Luck Bioscience CO. LTD, China).

MDA and ADMA levels were measured by the method of enzyme-linked immunosorbent assay. Commercial kits (KYM, China) were used. The standard substance and serum samples were incubated with MDA/ADMA antibody. Subsequently, the peroxidase-conjugated anti-human IgG was bound to the anti-MDA/ADMA antibodies. After coloration for $15 \mathrm{~min}$, the absorbance of each well was measured at $450 \mathrm{~nm}$ with an ultramark microplate reader (Bio-Rad, Hercules, CA). The concentration for each sample was calculated according to the standard curve, after subtraction of the blank values.

\section{Statistical analysis}

SPSS software version 17.0 (Statistical Package for the Social Sciences Inc., Chicago, IL, USA) was used to analyze all the data. Kolmogorov-Smirnov method was used to test whether the data was normal distributed. Variables were presented as mean \pm standard deviation, median with quartiles, or constituent ratio as appropriate. We used $t$ test for two independent samples or nonparametric test to compare the continuous variable and $x^{2}$ test to compare the categorical variable between groups. Pearson Correlation was used to analyze the correlation between variables. A $P$ value $<0.05$ was regarded as statistically significant.

\section{Results}

\section{Characteristics of study population}

Totally 35 patients with $\mathrm{PH}$ and 35 healthy controls were enrolled. Demographic data of the patients and healthy controls were presented in Table 1 . Among the $\mathrm{PH}$ patients, 12 patients were diagnosed with $\mathrm{PAH}$ (7 with idiopathic PAH, 4 with connective tissue diseases associated PAH, 1 with familial PAH), and 23 were diagnosed with CTEPH. The mean age was 44.6 years in PAH group and 55.0 years in CTEPH group. In PAH group, 2 patients (16.7\%) were male. In CTEPH group, 12 patients

Table 1 Characteristics of patients with PAH or CTEPH and healthy controls

\begin{tabular}{|c|c|c|c|c|c|c|}
\hline Variables & PAH group $(n=12)$ & PAH-Control $(n=12)$ & $P$ value & CTEPH group $(n=23)$ & CTEPH-Control $(n=23)$ & $P$ value \\
\hline Male, n (\%) & $2(16.7)$ & $2(16.7)$ & 1.000 & $12(52.2)$ & $11(47.8)$ & 0.768 \\
\hline Age, years & $44.6 \pm 13.1$ & $44.8 \pm 13.2$ & 0.963 & $55.0 \pm 11.6$ & $55.7 \pm 11.5$ & 0.839 \\
\hline BMI, $\mathrm{kg} / \mathrm{m}^{2}$ & $25.66 \pm 4.27$ & $25.12 \pm 3.73$ & 0.894 & $25.75 \pm 3.23$ & $24.53 \pm 3.01$ & 0.748 \\
\hline Hypertension, n (\%) & $2(16.7)$ & - & - & $5(21.7)$ & - & - \\
\hline Diabetes Mellitus, n (\%) & $0(0)$ & - & - & $1(4.3)$ & - & - \\
\hline 6MWD, m & $253.50(148.50,368.25)$ & - & - & $275.00(150.00,419.00)$ & - & - \\
\hline NT-proBNP, pg/ml & $1130.0(373.9,2312.0)$ & - & - & $1723.0(609.0,3136.0)$ & - & - \\
\hline $\mathrm{mPAP}, \mathrm{mmHg}$ & $50(37,55)$ & - & - & $52(49,55)$ & - & - \\
\hline PVRI, dyn $\cdot \mathrm{sec} \cdot \mathrm{m}^{2} / \mathrm{cm}^{5}$ & $1398.0(1149.0,2647.5)$ & - & - & $2231.5(1595.75,2611.5)$ & - & - \\
\hline $\mathrm{CO}, \mathrm{L} / \mathrm{min}$ & $3.95 \pm 1.21$ & - & - & $3.45 \pm 1.19$ & - & - \\
\hline $\mathrm{Cl}, \mathrm{L} / \mathrm{min} / \mathrm{m}^{2}$ & $2.27 \pm 0.67$ & - & - & $1.82 \pm 0.52$ & - & - \\
\hline
\end{tabular}

Data are presented as mean \pm SD or median (interquartile range). $\mathrm{PAH}=$ pulmonary arterial hypertension; $\mathrm{CTEPH}=\mathrm{Chronic}$ thromboembolic pulmonary hypertension; $\mathrm{BMI}=$ body mass index; $\mathrm{WHO}=$ World Health Organization; $6 \mathrm{MWD}=6$-minute walk distance; NT-proBNP $=\mathrm{N}$-terminal pro brain natriuretic peptide; $\mathrm{mPAP}=$ mean pulmonary arterial pressure; $\mathrm{PVRI}=$ pulmonary vascular resistance index; $\mathrm{CO}=$ cardiac output; $\mathrm{Cl}=\mathrm{cardiac}$ index. 
(52.2\%) patients were male. Most patients in PAH and CTEPH groups were WHO functional class II-III (74.9\% and $87.0 \%$, respectively). The comorbidity, 6MWD, level of NT-proBNP and main parameters of pulmonary haemodynamics were also presented in Table 1 . The median 6MWD was $253.50 \mathrm{~m}$ in PAH group and $275.00 \mathrm{~m}$ in CTEPH group, respectively.

\section{Comparison between $\mathrm{PAH}$ patients and $\mathrm{PAH}$-controls}

We compared the demographic characteristics and biomarkers level between $\mathrm{PAH}$ patients and PAH-controls (Table 1, Figure 1). The gender, age composition and BMI were well-matched. The mean levels of BAP and SOD were significantly lower in the PAH group compared with the control group $(P=0.014, P<0.001$, Figure $1 C$ and $D)$. Patients in PAH group had significantly higher ADMA level than the control group $(P=0.007$, Figure $1 \mathrm{E})$.

\section{Comparison between CTEPH patients and CTEPH-controls}

The demographic characteristics and biomarker levels were also compared between CTEPH patients and CTEPH-controls (Table 1, Figure 1). The mean level of MDA was significantly higher in CTEPH group $(P=0.034$, Figure 1B). The levels of BAP and SOD were obviously lower in the CTEPH group compared with the control group $(P=0.015, P<0.001$, Figure $1 C$ and $D)$. Patients in CTEPH group had significantly higher ADMA level than the control group $(P<0.001$, Figure $1 \mathrm{E})$.
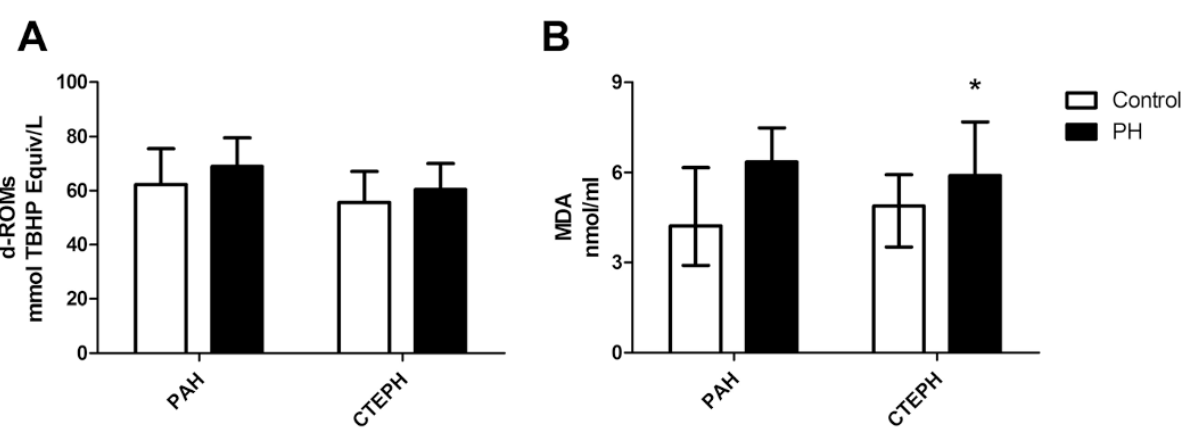

C

D
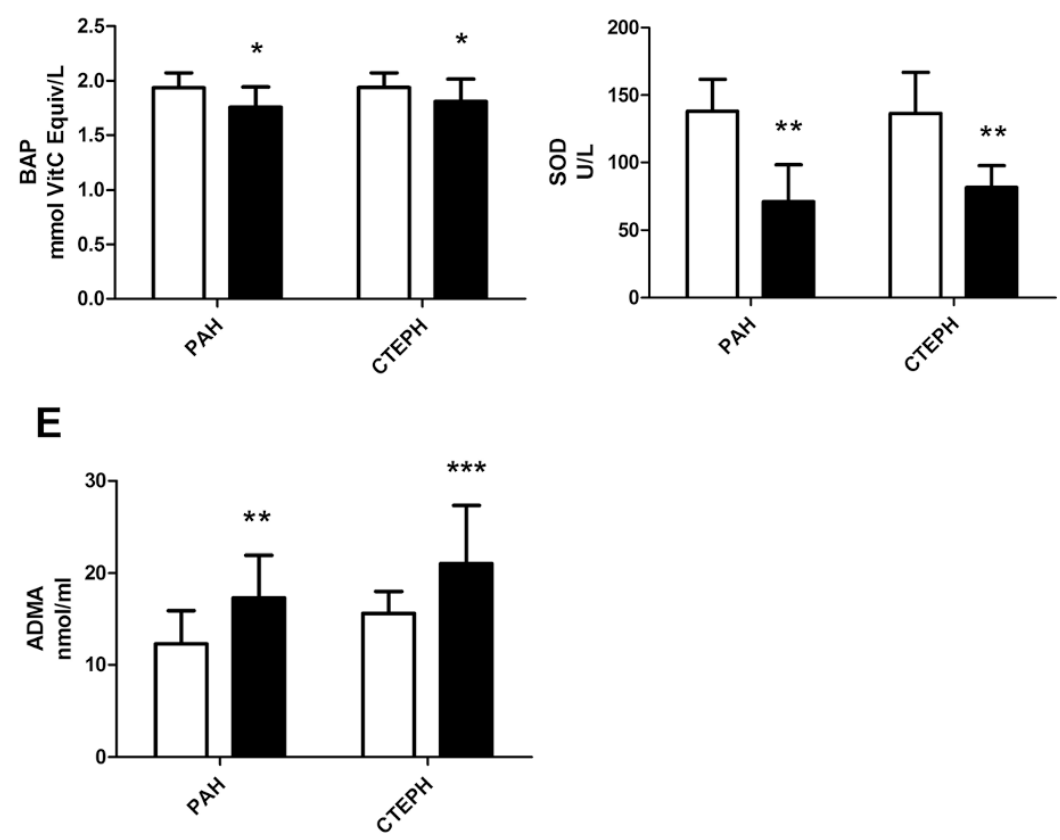

Figure 1 The comparison of oxidant-antioxidant biomarkers and ADMA levels. Data are presented as mean \pm standard deviation in Figure $\mathbf{A}, \mathbf{C}$ and $\mathbf{D}$, presented as median (interquartile range) in Figure $\mathbf{B}$. A. There was no significant difference in the level of d-ROMs between PAH group and PAH-control group, or between CTEPH and CTEPH-control group. B. The level of MDA was higher in PAH and CTEPH patients than that in their control group. The difference was only significant between CTEPH group and CTEPH-control group. $\mathbf{C}$. Compared with controls, the level of BAP was significantly lower in patients with PAH or CTEPH. D. Compared with controls, the level of SOD was significantly lower in patients with PAH or CTEPH. E. Compared with controls, the level of ADMA was significantly higher in patients with PAH or CTEPH. 
Table 2 The Correlation between Oxidant-antioxidant Biomarkers and ADMA

\begin{tabular}{llllll}
\hline Biomarkers & \multicolumn{2}{l}{ PAH } & & \multicolumn{2}{l}{ CTEPH } \\
\cline { 2 - 3 } & $\mathbf{r}$ & $\boldsymbol{P}$ & & $\mathbf{r}$ & $\boldsymbol{P}$ \\
\hline d-ROMs & -0.011 & 0.958 & & 0.151 & 0.333 \\
MDA & -0.017 & 0.958 & & 0.422 & 0.072 \\
BAP & -0.420 & 0.175 & & -0.333 & 0.164 \\
SOD & -0.436 & 0.156 & & 0.346 & 0.147 \\
\hline
\end{tabular}

$\mathrm{PAH}=$ pulmonary arterial hypertension; $\mathrm{CTEPH}=$ chronic thromboembolic pulmonary hypertension; $\mathrm{d}-\mathrm{ROMS}=$ derivative of reactive oxygen molecules; $\mathrm{MDA}=$ malondialdehyde; $\mathrm{BAP}=$ biological antioxidant potential; $\mathrm{SOD}=$ superoxide dismutase; $A D M A=$ asymmetric dimethylarginine.

\section{Correlation analysis}

We analyzed the correlation between the biomarkers (including d-ROMs, MDA, BAP, SOD and ADMA) and mPAP, PVRI, 6MWD, respectively, as well as the correlations between oxidative-antioxidative biomarkers and ADMA. The Pearson correlation coefficient and $P$ value were listed in Tables 2, 3, 4 and 5. No significant correlation was found between oxidative-antioxidative biomarkers and ADMA (Table 2). In patients with PAH, ADMA correlated positively with mPAP $(\mathrm{r}=0.762, P=0.006)$ and PVRI ( $=0.603, P=0.038)$ (Figure 2 ).

\section{Discussion}

\section{Oxidative-antioxidative status in $\mathrm{PH}$}

OS, an imbalance between ROS and antioxidant molecules, has been reported to be associated with various diseases, such as diabetes [26] and atherosclerosis [27]. Hypoxia, hypoperfusion, and instability in pulmonary circulation in PH may cause OS. In animal models, OS was found to be associated with the pathogenesis and development of PH [10]. However, few studies have explored the application of oxidative-antioxidative biomarkers in $\mathrm{PH}$ patients.

BAP and d-ROMs reflect the level of total antioxidants and oxidants in the samples, respectively. Previous studies

Table 3 The Correlation between the Biomarkers and mPAP

\begin{tabular}{llllll}
\hline Biomarkers & PAH & & & \multicolumn{2}{l}{ CTEPH } \\
\cline { 2 - 3 } & $\mathbf{r}$ & $\boldsymbol{P}$ & & $\mathbf{r}$ & $\boldsymbol{P}$ \\
\hline d-ROMs & 0.174 & 0.610 & & -0.273 & 0.231 \\
MDA & -0.107 & 0.754 & & 0.087 & 0.709 \\
BAP & -0.460 & 0.155 & -0.132 & 0.569 \\
SOD & -0.341 & 0.305 & & 0.118 & 0.611 \\
ADMA & 0.762 & $0.006^{*}$ & -0.097 & 0.700
\end{tabular}

$\mathrm{mPAP}=$ mean pulmonary arterial pressure; $\mathrm{PAH}=$ pulmonary arterial hypertension; $\mathrm{CTEPH}=$ chronic thromboembolic pulmonary hypertension; $\mathrm{d}$-ROMS = derivative of reactive oxygen molecules; $\mathrm{MDA}=$ malondialdehyde; $\mathrm{BAP}=$ biological antioxidant potential; $\mathrm{SOD}=$ superoxide dismutase; ADMA = asymmetric dimethylarginine.

*The difference with control is statistically significant.
Table 4 The Correlation between the Biomarkers and PVRI

\begin{tabular}{llllll}
\hline Biomarkers & \multicolumn{2}{l}{ PAH } & & \multicolumn{2}{l}{ CTEPH } \\
\cline { 2 - 3 } & $\mathbf{r}$ & $\boldsymbol{P}$ & & $\mathbf{r}$ & $\boldsymbol{P}$ \\
\hline d-ROMs & 0.352 & 0.353 & & -0.410 & 0.115 \\
MDA & 0.022 & 0.956 & & 0.266 & 0.320 \\
BAP & -0.581 & 0.101 & -0.491 & 0.053 \\
SOD & -0.654 & 0.056 & & 0.043 & 0.875 \\
ADMA & 0.603 & $0.038^{*}$ & -0.097 & 0.700
\end{tabular}

$\mathrm{PVRI}=$ pulmonary vascular resistance index $; \mathrm{PAH}=$ pulmonary arterial hypertension; $\mathrm{CTEPH}=$ chronic thromboembolic pulmonary hypertension; $\mathrm{d}$-ROMS = derivative of reactive oxygen molecules; $\mathrm{MDA}=$ malondialdehyde; $\mathrm{BAP}=$ biological antioxidant potential; $\mathrm{SOD}=$ superoxide dismutase; ADMA = asymmetric dimethylarginine.

*The difference is statistically significant.

found that d-ROMs level was high in COPD patients [28], and was associated with the severity of IPF [20]. BAP level was significantly lower in patients with metabolic syndrome [29], and was closely associated with diabetic retinopathy and nephropathy in patients with type 2 diabetes [30].

The most possible reason for decreased BAP and SOD level in patients with $\mathrm{PH}$ is the consumption of antioxidant substances in the OS. The level of d-ROMs stayed normal in $\mathrm{PAH}$ and CTEPH, which was possibly in compensatory status. The finding of our study suggests that OS contributes to the pathogenesis of $\mathrm{PAH}$ and CTEPH. In animal models, SOD augmentation regresses experimental PAH [31]. The antioxidant therapy might be used as a supplement in the treatment of $\mathrm{PH}$.

In a group of 347 patients who underwent echocardiographic assessment, the relationship between pulmonary artery systolic pressure (PASP) measured by echocardiography and plasma aminothiol OS markers was investigated. For each $1 \%$ increase in plasma cystine, PASP increased by $16 \%$ [32]. However, in our current study, we did not find correlation between oxidative-antioxidative biomarkers and mPAP or PVRI measured in right heart

Table 5 The Correlation between the Biomarkers and 6MWD

\begin{tabular}{llllll}
\hline Biomarkers & PAH & & & \multicolumn{2}{l}{ CTEPH } \\
\cline { 2 - 3 } & $\mathbf{r}$ & $\boldsymbol{P}$ & & $\mathbf{r}$ & $\boldsymbol{P}$ \\
\hline d-ROMs & -0.357 & 0.386 & & -0.092 & 0.765 \\
MDA & -0.071 & 0.867 & & -0.311 & 0.301 \\
BAP & 0.546 & 0.162 & -0.166 & 0.588 \\
SOD & 0.548 & 0.159 & & 0.477 & 0.100 \\
ADMA & -0.454 & 0.259 & -0.369 & 0.264 \\
\hline
\end{tabular}

$6 \mathrm{MWD}=6$-minute walk distance; $\mathrm{PAH}=$ pulmonary arterial hypertension; $\mathrm{CTEPH}=$ chronic thromboembolic pulmonary hypertension; $\mathrm{d}-\mathrm{ROMS}=$ derivative of reactive oxygen molecules; $\mathrm{MDA}=$ malondialdehyde; $\mathrm{BAP}=$ biological antioxidant potential; $S O D=$ superoxide dismutase; $A D M A=$ asymmetric dimethylargininenn. 


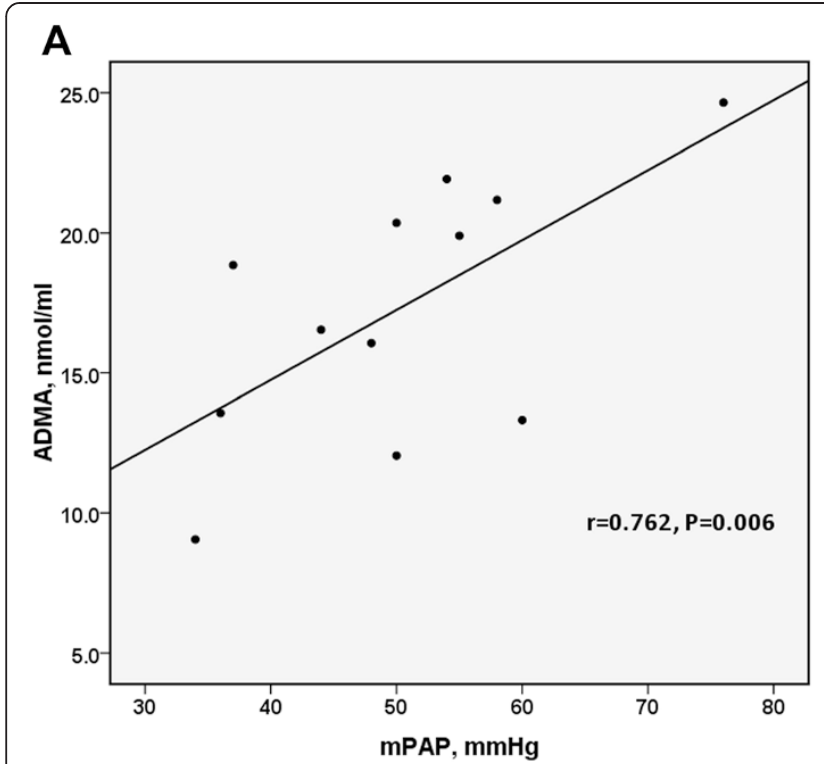

\section{B}

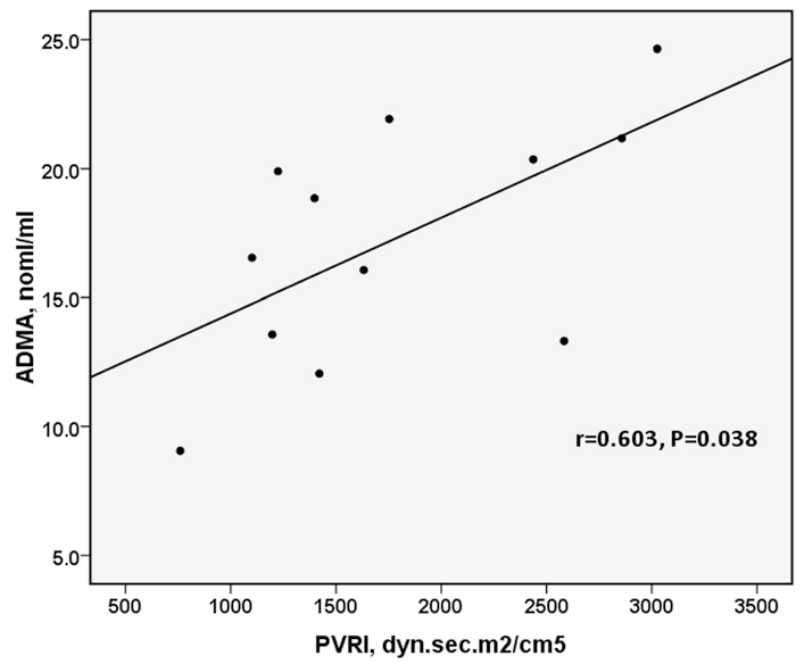

Figure 2 The correlation between ADMA and mPAP, PVRI in patients with PAH. In patients with PAH, mPAP $(r=0.762, P=0.006)$ and PVRI $(r=0.603, P=0.038)$ were both positively associated with the level of ADMA.

catheterization. One possible reason is that we used different OS biomarkers and different procedures to measure pulmonary artery pressure. Another possible reason is the limited sample size of our study. Right heart catheterization is the golden standard for $\mathrm{PH}$ diagnosis and mPAP is more accurate than PASP estimated in echocardiography. The relationship between OS biomarkers and pulmonary artery pressure needs to be explored in more patients who undergo right heart catheterization assessment.

\section{Increased ADMA in PH and its significance}

ADMA is a natural ubiquitous amino acid. The lungs are a major source of $\mathrm{NO}$ synthase and ADMA, which are in a dynamic balance and determine NO production [33]. ADMA has multiple functions, such as vasoconstriction, impairing endothelial-related dilatation, and increasing endothelial adhesivity [34]. Plasma ADMA levels are related with endothelial dysfunction. ADMA levels are elevated in many cardiovascular and metabolic diseases such as coronary artery disease [35] and hypertension [36]. In our study, ADMA level was elevated in PH, which is consistent with previous studies [17,18,37-39]. The potential mechanism that ADMA contributes to $\mathrm{PH}$ has been studied recently. ADMA is metabolized by dimethyl-arginine dimethylaminohydrolase. Suppression of endothelial dimethyl-arginine dimethylaminohydrolase expression and function represents as an important underlying mechanism in hypoxia-induced $\mathrm{PH}$ [40] and IPAH [41]. ADMA may induce pulmonary endothelial dysfunction via changes in the expression and activity of connexin 43 [42].
Also in our current study, the serum ADMA level correlated positively with mPAP in $\mathrm{PAH}$ patients, as well as PVRI. In previous studies, plasma ADMA concentrations correlated with mixed venous saturation, right atrial pressure, and cardiac index [18]. These findings indicate that the down-regulation of NO/cGMP pathway plays a crucial role in $\mathrm{PAH}$, and the inhibition degree of this pathway affects the disease severity. The possible explanation for this association is that the NO/cGMP pathway regulates pulmonary vascular tone, which can be reflected directly by MPAP and PVRI. The pathophysiological role of ADMA in PAH still remains to be investigated. The relationship between the ADMA and NO system is probably more complicated than currently known. Since ADMA correlates with multiple parameters of pulmonary hemodynamics, it may be useful in the evaluation of disease severity, assessment of therapeutic efficiency, and risk stratification in patients with $\mathrm{PAH}$ or other kinds of PH. Given the small sample size of our study, further studies are needed to explore the values of ADMA in PH.

\section{Relationship between oxidative-antioxidative biomarkers and ADMA}

Accumulating evidences have demonstrated that $\mathrm{NO}$ is closely associated with ROS. During chronic hypoxia, increased vascular superoxide anion production adversely impact endothelial function by impairing NO signaling [43]. When enough ROS are produced, they will start reacting readily with $\mathrm{NO}$ to form the peroxynitrite [44]. $\mathrm{NO}$, the endothelium-derived relaxing factor, decreases smooth muscle metabolism and reduces ROS production. 
ADMA increases intracellular ROS generation in bovine retinal capillary endothelial cells [45]. In this study, we did not find association between oxidative-antioxidative biomarkers and ADMA in peripheral serum. The level of these biomarkers in peripheral circulation is a reflection of overall production and cleavage of ROS, RNS, and $\mathrm{NO}$, which is more complicated and may be affected by various factors.

The pathogenesis of $\mathrm{PH}$ is a complex multifactorial process. OS and impaired NO/sGC/cGMP signal pathway are both involved, and their relationship is complicated. We speculate that ROS increase first, then NO decreases. The three major stimuli that drive the vascular remodeling process are inflammation, shear stress and hypoxia. All these stimuli may increase the production of ROS, which leads to low levels of tetrahydrobiopterin and L-arginine, the rate limiting co-factor and substrate for endothelial NO synthase. Then endothelial NO synthase is uncoupled, resulting in decreased NO production and increased ROS production in turn [9]. We think that this process happens rapidly in our body, therefore, we couldn't detect the change of oxidative-antioxidative biomarkers before the change of NO signaling biomarkers like ADMA and vice versa.

\section{Conclusions}

In summary, the antioxidative potential decreases while serum ADMA is elevated in PAH and CTEPH patients. Increased ADMA serum levels are associated with unfavorable pulmonary hemodynamics in PAH patients. ADMA may be useful in the severity evaluation and risk stratification of PAH.

\section{Ethics statement}

The study protocol was approved by the Ethics Committee of Beijing Hospital (2014BJYYEC-051-01). Informed consent was obtained from each subject.

\section{Abbreviations}

OS: Oxidative stress; NO: Nitric oxide; PH: Pulmonary hypertension; PAH: Pulmonary arterial hypertension; CTEPH: Chronic thromboembolic pulmonary hypertension; mPAP: Mean pulmonary artery pressure; PVRI: Pulmonary vascular resistance index; $d$-ROMs: Derivative of reactive oxygen molecules; BAP: Biological antioxidant potential; SOD: Superoxide dismutase; MDA: Malondialdehyde; ADMA: Asymmetric dimethylarginine; WHO: World Health Organization; OS: Oxidative stress; ROS: Reactive oxygen species; RNS: Reactive nitrogen species; IPAH: Idiopathic pulmonary arterial hypertension; 6MWD: Six minute walk distance; TBHP: Tert butyl hydroperoxide; VitC: Vitamin C; PASP: Pulmonary artery systolic pressure.
}

\section{Competing interests}

The authors declare that they have no competing interests.

\section{Authors' contributions}

$S Z$ and $C W$ had full access to all of the data in the study and take responsibility for the integrity of the data and the accuracy of the data analysis. SZ: contributed to the study design, data acquisition and collection; sample collection and biomarkers measurement; data analysis and interpretation, and manuscript preparation and revision. TY and XX: contributed to the study design; data analysis and interpretation; and manuscript preparation, revision, and approval of the final version. $M W$ : contributed to the method establishment and evaluation; biomarkers measurement; and manuscript preparation, revision, and approval of the final version. $L Z$ : contributed to the method establishment and evaluation; data acquisition and collection; sample collection and biomarkers measurement; and data analysis, and approval of the final version. YY: contributed to the study design; patients enrollment and assessment; data interpretation; and manuscript preparation, revision, and approval of the final version. ZZ: contributed to the study design; patients enrollment and assessment; data interpretation; and manuscript preparation, revision, and approval of the final version. FX: contributed to the study design; data analysis and interpretation; and manuscript preparation, revision, and approval of the final version. CW: contributed to the study design; data interpretation; and manuscript preparation, revision, and approval of the final version. All authors read and approved the final manuscript.

\section{Funding}

This work was supported by National Key Technology Research and Development Program, [Grant2011BAl11B17]; The Capital Health Research and Development Special Fund, [2011-4011-05]; National Key Technology Research and Development Program, [Grant 2012BAI05B02]; National High Technology Research and Development Program Grant 2012AA02A511].

\section{Author details}

${ }^{1}$ Department of Pulmonary and Critical Care Medicine, Beijing Institute of Respiratory Medicine, Beijing Chao-Yang Hospital, Capital Medical University, Beijing, People's Republic of China. ${ }^{2}$ Beijing Key Laboratory of Respiratory and Pulmonary Circulation Disorders, Beijing, People's Republic of China. ${ }^{3}$ Department of Pulmonary and Critical Care Medicine, Beijing Hospital, Beijing, People's Republic of China. ${ }^{4}$ National Clinical Research Center of Respiratory Diseases, Beijing, People's Republic of China. ${ }^{5}$ Department of Laboratory Medicine, Beijing Hospital, Beijing, People's Republic of China. ${ }^{6}$ Department of Cell Biology, Institute of Geriatrics, Beijing Hospital, Beijing, People's Republic of China. ${ }^{7}$ Department of Respiratory Medicine, Capital Medical University, Beijing, People's Republic of China. ${ }^{8}$ China-Japan Friendship Hospital, Beijing, People's Republic of China.

Received: 24 November 2014 Accepted: 22 April 2015

Published online: 02 May 2015

\section{References}

1. Galie N, Hoeper MM, Humbert M, Torbicki A, Vachiery JL, Barbera JA, et al. Guidelines for the diagnosis and treatment of pulmonary hypertension: the Task Force for the Diagnosis and Treatment of Pulmonary Hypertension of the European Society of Cardiology (ESC) and the European Respiratory Society (ERS), endorsed by the International Society of Heart and Lung Transplantation (ISHLT). Eur Heart J. 2009;30(20):2493-537.

2. Archer $\mathrm{S}$, Rich S. Primary pulmonary hypertension: a vascular biology and translational research "Work in progress". Circulation. 2000;102(22):2781-91.

3. Grobe AC, Wells SM, Benavidez E, Oishi P, Azakie A, Fineman JR, et al. Increased oxidative stress in lambs with increased pulmonary blood flow and pulmonary hypertension: role of NADPH oxidase and endothelial NO synthase. Am J Physiol Lung Cell Mol Physiol. 2006;290(6):L1069-77.

4. Karuppiah K, Druhan L, Chen CA, Smith T, Zweier JL, Sessa WC, et al. Suppression of eNOS-derived superoxide by caveolin-1: a biopterin-dependent mechanism. Am J Physiol Lung Cell Mol Physiol. 2011;301(3):H903-11.

5. Birukov KG. Cyclic stretch, reactive oxygen species, and vascular remodeling. Antioxid Redox Signaling. 2009;11(7):1651-67.

6. DeMarco VG, Habibi J, Whaley-Connell AT, Schneider RI, Heller RL, Bosanquet JP, et al. Oxidative stress contributes to pulmonary hypertension in the transgenic (mRen2)27 rat. Am J Physiol Lung Cell Mol Physiol. 2008;294(6):H2659-68.

7. Rawat DK, Alzoubi A, Gupte R, Chettimada S, Watanabe M, Kahn AG, et al. Increased reactive oxygen species, metabolic maladaptation, and autophagy contribute to pulmonary arterial hypertension-induced ventricular hypertrophy and diastolic heart failure. Hypertension. 2014;64(6):1266-74.

8. Iwata K, Ikami K, Matsuno K, Yamashita T, Shiba D, Ibi M, et al. Deficiency of NOX1/nicotinamide adenine dinucleotide phosphate, reduced form oxidase leads to pulmonary vascular remodeling. Arterioscler Thromb Vasc Biol. 2014;34(1):110-9. 
9. Aggarwal S, Gross CM, Sharma S, Fineman JR, Black SM. Reactive oxygen species in pulmonary vascular remodeling. Comprehensive Physiology. 2013;3(3):1011-34.

10. Villegas LR, Kluck D, Field C, Oberley-Deegan RE, Woods C, Yeager ME, et al. Superoxide dismutase mimetic, MnTE-2-PyP, attenuates chronic hypoxia-induced pulmonary hypertension, pulmonary vascular remodeling, and activation of the NALP3 inflammasome. Antioxid Redox Signaling. 2013;18(14):1753-64.

11. Fan YF, Zhang $R$, Jiang $X$, Wen $L, W u$ DC, Liu D, et al. The phosphodiesterase-5 inhibitor vardenafil reduces oxidative stress while reversing pulmonary arterial hypertension. Cardiovasc Res. 2013;99(3):395-403.

12. Cooke JP. ADMA: its role in vascular disease. Vascular medicine (London, England). 2005;10 Suppl 1:S11-7.

13. Miyazaki H, Matsuoka H, Cooke JP, Usui M, Ueda S, Okuda S, et al. Endogenous nitric oxide synthase inhibitor: a novel marker of atherosclerosis. Circulation. 1999;99(9):1141-6.

14. Fujiwara N, Osanai T, Kamada T, Katoh T, Takahashi K, Okumura K. Study on the relationship between plasma nitrite and nitrate level and salt sensitivity in human hypertension : modulation of nitric oxide synthesis by salt intake. Circulation. 2000;101(8):856-61.

15. Stuhlinger MC, Abbasi F, Chu JW, Lamendola C, McLaughlin TL, Cooke JP, et al. Relationship between insulin resistance and an endogenous nitric oxide synthase inhibitor. JAMA. 2002;287(11):1420-6.

16. Notsu Y, Yano S, Shibata H, Nagai A, Nabika T. Plasma arginine/ADMA ratio as a sensitive risk marker for atherosclerosis: Shimane CoHRE study. Atherosclerosis. 2015;239(1):61-6.

17. Kielstein JT, Bode-Boger SM, Hesse G, Martens-Lobenhoffer J, Takacs A, Fliser $D$, et al. Asymmetrical dimethylarginine in idiopathic pulmonary arterial hypertension. Arterioscler Thromb Vasc Biol. 2005;25(7):1414-8.

18. Skoro-Sajer N, Mittermayer F, Panzenboeck A, Bonderman D, Sadushi R, Hitsch $R$, et al. Asymmetric dimethylarginine is increased in chronic thromboembolic pulmonary hypertension. Am J Respir Crit Care Med. 2007;176(11):1154-60.

19. Komatsu F, Kudoh H, Kagawa Y. Evaluation of oxidative stress and effectiveness of low-dose glucocorticoid therapy on exacerbation of chronic obstructive pulmonary disease. J Gerontol A: Biol Med Sci. 2007;62(4):459-64.

20. Daniil ZD, Papageorgiou E, Koutsokera A, Kostikas K, Kiropoulos T, Papaioannou Al, et al. Serum levels of oxidative stress as a marker of disease severity in idiopathic pulmonary fibrosis. Pulm Pharmacol Ther. 2008;21(1):26-31.

21. Moore K, Roberts 2nd L. Measurement of lipid peroxidation. Free Radic Res. 1998;28(6):659-71.

22. Del Rio D, Stewart AJ, Pellegrini N. A review of recent studies on malondialdehyde as toxic molecule and biological marker of oxidative stress. Nutr Metab Cardiovasc Dis. 2005;15(4):316-28.

23. Ismail M, Hossain MF, Tanu AR, Shekhar HU. Effect of spirulina intervention on oxidative stress, antioxidant status, and lipid profile in chronic obstructive pulmonary disease patients. BioMed research international 2015. 2015;2314-6141(Electronic):486120.

24. Margaritelis NV, Veskoukis AS, Paschalis V, Vrabas IS, Dipla K, Zafeiridis A, et al. Blood reflects tissue oxidative stress: a systematic review. Biomarkers: biochemical indicators of exposure, response, and susceptibility to chemicals. 2015;1366-5804(Electronic):1-12.

25. Hoeper MM, Bogaard HJ, Condliffe R, Frantz R, Khanna D, Kurzyna M, et al. Definitions and diagnosis of pulmonary hypertension. J Am Coll Cardiol. 2013;62(25 Suppl):D42-50.

26. Curtis JM, Grimsrud PA, Wright WS, Xu X, Foncea RE, Graham DW, et al. Downregulation of adipose glutathione S-transferase A4 leads to increased protein carbonylation, oxidative stress, and mitochondrial dysfunction. Diabetes. 2010;59(5):1132-42.

27. Manea A, Simionescu M. Nox enzymes and oxidative stress in atherosclerosis. Front Biosci (Schol Ed). 2012;4:651-70.

28. Markoulis N, Gourgoulianis Kl, Moulas A, Gerogianni E, Molyvdas AP. Reactive oxygen metabolites as an index of chronic obstructive pulmonary disease severity. Panminerva Med. 2006;48(4):209-13.

29. Kim JH, Baik HW, Yoon YS, Joung HJ, Park JS, Park SJ, et al. Measurement of antioxidant capacity using the biological antioxidant potential test and its role as a predictive marker of metabolic syndrome. Korean J Intern Med. 2014;29(1):31-9.

30. Naruse R, Suetsugu M, Terasawa T, Ito K, Hara K, Takebayashi K, et al. Oxidative stress and antioxidative potency are closely associated with diabetic retinopathy and nephropathy in patients with type 2 diabetes. Saudi Med J. 2013;34(2):135-41.

31. Archer SL, Marsboom G, Kim GH, Zhang HJ, Toth PT, Svensson EC, et al. Epigenetic attenuation of mitochondrial superoxide dismutase 2 in pulmonary arterial hypertension: a basis for excessive cell proliferation and a new therapeutic target. Circulation. 2010;121(24):2661-71.

32. Ghasemzadeh N, Patel RS, Eapen DJ, Veledar E, Al Kassem H, Manocha P, et al. Oxidative stress is associated with increased pulmonary artery systolic pressure in humans. Hypertension. 2014;63(6):1270-5.

33. Bulau P, Zakrzewicz D, Kitowska K, Leiper J, Gunther A, Grimminger F, et al. Analysis of methylarginine metabolism in the cardiovascular system identifies the lung as a major source of ADMA. Am J Physiol Lung Cell Mol Physiol. 2007;292(1):L18-24.

34. Vallance $P$, Leiper J. Cardiovascular biology of the asymmetric dimethylarginine:dimethylarginine dimethylaminohydrolase pathway. Arterioscler Thromb Vasc Biol. 2004;24(6):1023-30.

35. Plicner D, Mazur P, Sadowski J, Undas A. Asymmetric dimethylarginine and oxidative stress following coronary artery bypass grafting: associations with postoperative outcome. European journal of cardio-thoracic surgery: official journal of the European Association for Cardio-thoracic Surgery. 2014;45(5):e136-41.

36. Fan NC, Tsai CM, Hsu CN, Huang LT, Tain YL. N-acetylcysteine prevents hypertension via regulation of the ADMA-DDAH pathway in young spontaneously hypertensive rats. BioMed research international. 2013;2013:696317.

37. Trittmann JK, Peterson E, Rogers LK, Chen B, Backes CH, Klebanoff MA, et al. Plasma asymmetric dimethylarginine levels are increased in neonates with bronchopulmonary dysplasia-associated pulmonary hypertension. J Pediatr. 2015;166(2):230-3.

38. Giannakoulas G, Mouratoglou SA, Gatzoulis MA, Karvounis H. Blood biomarkers and their potential role in pulmonary arterial hypertension associated with congenital heart disease. a systematic review. Int J Cardiol. 2014;174(3):618-23.

39. Parikh RV, Scherzer R, Nitta EM, Leone A, Hur S, Mistry V, et al. Increased levels of asymmetric dimethylarginine are associated with pulmonary arterial hypertension in HIV infection. Aids. 2014;28(4):511-9.

40. Millatt L, Whitley GS, Li D, Leiper JM, Siragy HM, Carey RM, et al. Evidence for dysregulation of dimethylarginine dimethylaminohydrolase I in chronic hypoxia-induced pulmonary hypertension. Circulation. 2003;108(12):1493-8.

41. Pullamsetti S, Kiss L, Ghofrani HA, Voswinckel R, Haredza P, Klepetko W, et al. Increased levels and reduced catabolism of asymmetric and symmetric dimethylarginines in pulmonary hypertension. FASEB journal : official publication of the Federation of American Societies for Experimental Biology. 2005;19(9):1175-7.

42. Tsang H, Leiper J, Hou Lao K, Dowsett L, Delahaye MW, Barnes G, et al. Role of asymmetric methylarginine and connexin 43 in the regulation of pulmonary endothelial function. Pulmonary circulation. 2013;3(3):675-91.

43. Houston M, Estevez A, Chumley P, Aslan M, Marklund S, Parks DA, et al. Binding of xanthine oxidase to vascular endothelium. Kinetic characterization and oxidative impairment of nitric oxide-dependent signaling The Journal of biological chemistry. 1999;274(8):4985-94.

44. Kamezaki F, Tasaki H, Yamashita K, Tsutsui M, Koide S, Nakata S, et al. Gene transfer of extracellular superoxide dismutase ameliorates pulmonary hypertension in rats. Am J Respir Crit Care Med. 2008;177(2):219-26.

45. Chen YH, Xu X, Sheng MJ, Zheng Z, Gu Q. Effects of asymmetric dimethylarginine on bovine retinal capillary endothelial cell proliferation, reactive oxygen species production, permeability, intercellular adhesion molecule-1, and occludin expression. Mol Vis. 2011;17:332-40. 